\title{
Real-Time Implementation of DC Servomotor Actuator with Unknown Uncertainty using a Sliding Mode Observer
}

\author{
Roxana-Elena Tudoroiu \\ University of Petrosani
}

20 Universităţii street, 332006, Petroșani, Hunedoara, Romania Email: tudelena@mail.com

\section{Nicolae Ilias University of Petrosani}

20 Universității street, 332006, Petroșani, Hunedoara, Romania Email: iliasnic@yahoo.com

\author{
Wilhelm Kecs \\ University of Petrosani
}

20 Universităţii street, 332006,

Petroșani, Hunedoara, Romania

Email: wwkecs@yahoo.com

\section{Stelian-Valentin Casavela University of Petrosani}

20 Universității street, 332006, Petroșani, Hunedoara, Romania Email: svcasavela@yahoo.com

\author{
Maria Dobritoiu \\ University of Petrosani
}

20 Universităţii street, 332006,

Petroșani, Hunedoara, Romania

Email:mariadobritoiu@yahoo.com

Nicolae Tudoroiu

John Abbott College 2127

Lakeshore Road, Sainte-Anne-de-

Bellevue, QC, H9X 3L9, Canada

Email: ntudoroiu@gmail.com

\begin{abstract}
The central idea of this paper is the modeling and implementation of a real-time dc servomotor angular speed control system with an unknown bounded uncertainty using a sliding mode observer (SMO) control strategy. We prefer to use a SMO in our approach due to its great potential in fault detection and isolation (FDI) of the actuators and sensor faults subjected frequently to several failures due to an abnormal change in their operating conditions or parameters. We use for this purpose the most suitable real time implementation tool MATLAB/SIMULINK software package. It provides special features for real time implementation by its extensions RealTime Workshop (RTW) and the Real-Time Windows Target (RTWT). The novelty of our paper is to prove in an extensive simulation MATLAB/SIMULINK frame the real time implementation potential of a most recently sliding mode observer (SMO) control strategy applied to a particular case study, namely for a de servomotor angular speed control system. The proposed real-time Sliding Mode Observer (SMO) consists of an embedded nonlinear Sliding Mode Observer (SMO) with the de servomotor actuator in an integrated control system structure to estimate its angular speed and armature current and to implement the sliding mode control law.
\end{abstract}

\section{INTRODUCTION}

$\mathrm{T}$ HE most important common actuator integrated in a forward path of the control systems structure is the dc servomotor. It directly provides rotary motion and, coupled with wheels or drums and cables, can also provide transitional motion. The cause roots of the majority faults in control systems are the result of unexpected failures, interferences as well as the age of their crucial components. In our research defective measurement and control loops equipment, in particular a sensor and an actuator are under investigation. More precisely, we assume that the dc servomotor control system is subjected to an unknown but bounded uncertainty, and its speed is controlled in closed-loop feedback by using a state feedback control law with an embedded sliding mode observer (SMO) that is integrated in the closed-loop control structure. The dc servomotors are preferred in control area applications because they possess a high start torque characteristics, high response performance, and easier to be linearly controlled etc. Also the dc servomotor speed can be adjusted by varying its input voltage. Therefore, the dc motor control is better compared to all ac induction motors that need expensive frequency drivers. The real-time dc servomotor speed control can be easily understood and interfaced with MATLAB/SIMULINK or AnyLogic multi-paradigms hybrid simulator in the case of UML-RT implementations [8] with a fast response. Furthermore, the preliminary results of this research will be useful for us for future exploration of using a sliding mode observer to develop more attractive control strategies, for example the detection and isolation of the faults (FDI) [1], [5], [6], [7] that occur in the actuators and sensors, and also for trust modeling in Multi-agent Systems [3], will be an interesting future directions of our research. Whenever these critical situations come out the control systems could lose the control, require much more energy, and could operate harmfully. Therefore to operate in real-time at high energy efficiency and to guarantee the equipment safety and reliability it is important to develop suitable FDI strategies capable to detect and diagnose any time every faulty control system components and consequently corrective and reconfiguration actions should be initiated promptly [7]. Effectively the existing methods to identify and to adjust the equipment failures are mostly labor-intensive task, and consequently sustained, rhythmic and error-prone [7]. In the majority of these situations the windings currents are recorded to determine the health of the dc servomotors currents compared to statistical evaluation that necessitates considerable human knowledge, hence error-prone that could generate severely equipment operation. In these conditions the problem of control systems monitoring and fault diagnosis becomes a critical issue, of high complexity that need to be 
implemented in mainframe environment by using more sophisticated control systems and artificial intelligence strategies. This research work is based on our previous results in implementation of different control systems strategies and now we are interested to prove the effectiveness of real-time implementation of our proposed SMO control strategy. Closing, the main objective of our research is to develop a more suitable, accurate and consistent real-time nonlinear SMO control strategy to be used in our future real-time FDI control strategies implementation development.

\section{THE DC SERVOMOTOR DYNAMICS}

The electric circuit of the dc servomotor armature and the free body diagram of the rotor are shown in figure 1. For simulation purpose we will assume the following experimental values for the physical parameters [9]:

- moment of inertia of the rotor: $J=0.001\left[\frac{\mathrm{kgm}^{2}}{\mathrm{~s}^{2}}\right]$

- damping ratio of the mechanical system: $b=0.01[\mathrm{Nms}]$

- counter electromotive force coefficient (cemf):

$$
\mathrm{k}_{\mathrm{e}}=\mathrm{k}_{\mathrm{t}}=\mathrm{k}=0.0517[\mathrm{Nm} / \mathrm{A}]
$$

- motor electric resistance: $\mathrm{R}=1[\Omega]$

- motor electric inductance: $\mathrm{L}=0.5[\mathrm{H}]$

- motor initial angular speed: $\omega=1\left[\frac{\mathrm{rad}}{\mathrm{s}}\right]$,

- $\quad$ input de power supply: $V=12[\mathrm{~V}]$

- load torque: $T_{L}=0.1 \sin (t)[N m]$

The dynamics of the dc servomotor actuator is described by the following input-state-output equations [8]-[9] :

$$
\begin{aligned}
& J \frac{d^{2} \theta}{d t^{2}}+b \frac{d \theta}{d t}=k_{t} I_{a}-T_{L} \\
& L \frac{d I_{a}}{d t}+R I_{a}=V-k_{e} \frac{d \theta}{d t} .
\end{aligned}
$$

where $T_{e}=T=k_{t} I_{a}$ is the dc servomotor torque developed to the shaft, $T_{L}$ is the load torque, and $e=k_{e} \frac{d \theta}{d t}=k_{e} \omega$ is the counter electromotive force (cemf) of the dc servomotor.

In a state-space representation the dc servomotor actuator dynamics is described by following equations:

(i) State Equation:

$$
\begin{gathered}
{\left[\begin{array}{c}
\frac{d x_{1}}{d t} \\
\frac{d x_{2}}{d t}
\end{array}\right]=\left[\begin{array}{cc}
-\frac{b}{J} & \frac{k_{t}}{J} \\
-\frac{k_{e}}{L} & -\frac{R}{L}
\end{array}\right]\left[\begin{array}{l}
x_{1} \\
x_{2}
\end{array}\right]+\left[\begin{array}{c}
-\frac{T_{L}}{J} \\
\frac{u}{L}
\end{array}\right]} \\
x_{1}=\omega, x_{2}=I_{a}, u=V, x_{1}(0)=1\left[\frac{\mathrm{rad}}{\mathrm{s}}\right], x_{2}(0)=0[A]
\end{gathered}
$$

(ii) Output equation:

$$
y=\left[\begin{array}{ll}
1 & 0
\end{array}\right]\left[\begin{array}{l}
x_{1} \\
x_{2}
\end{array}\right]
$$

where we assume that the only the state $x_{1}$ is measurable, and the input command $u=V$ is a function defined by the armature voltage (dc power supply) and is designed in closed-loop as a state feedback control law. Also the load torque $T_{L}$ is considered unknown which is bounded and has a bounded derivative.
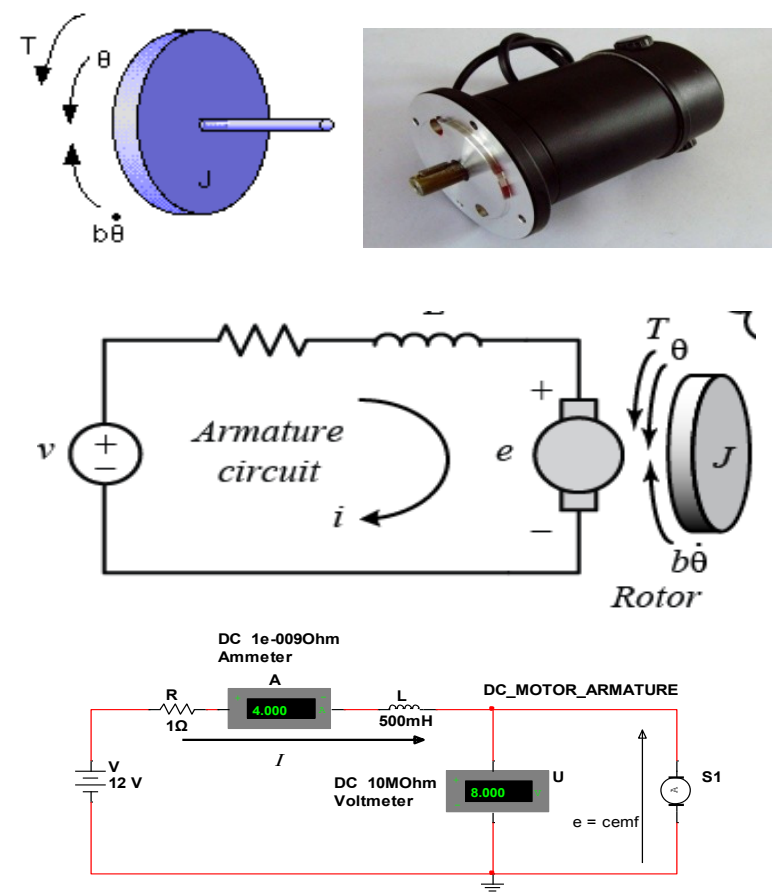

Fig. 1 The simplified equivalent electrical circuit of the dc servomotor (Reproduced from [8]-[9])

\section{SLIDING MODE CONTROLLERS VERSUS SLIDING MODE OBSERVERS}

To formulate any practical control application is not a straightforward task due to a more or less mismatch between the actual plant and its mathematical model used for the controller design. This mismatch could arise from unknown external disturbances, plant parameters, or modeling errors. To design a suitable control law in order to provide the desired performance to the negative feedback closed-loop control system in the presence of these disturbances or uncertainties is a very difficult task for any control engineer. This has led to an intensive research to develop some kind of robust control methods that are supposed to solve this problem. The sliding mode control (SMC) is one of particular attractive approach to design a robust controller due to its potential as a robust control method. A sliding mode control (SMC) is characterized by a suite of feedback control laws and a decision rule [1]. The decision rule is a sort of switching function that has as its input some measure of the current system behavior in order to generate as an output a particular feedback robust controller which should be used at that time instant [1]-[2]. The sliding mode control (SMC) strategy is designed such that the variable structure control systems have to be capable to drive the system states on the convergent phase trajectories and then to constrain these to lie within a neighborhood of the switching function 
that can be represented by a switching line or switching surface, such is shown in figure 2 [8]. In this approach the dynamic behavior of the control system may be adapted to a particular choice of switching function, and also the feedback closed-loop response becomes totally insensitive to a particular class of uncertainty in the system, more precisely proving a high robustness feature to all kind of system uncertainties. The main drawback of using the sliding mode control (SMC) techniques to design a large variety of control industrial applications is the necessity to implement a basically discontinuous control signal which hypothetically must switch with infinite frequency to provide total rejection of the uncertainties [1]-[2].

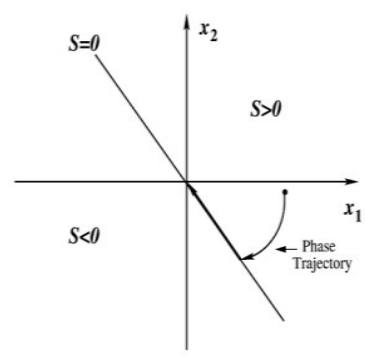

(a)

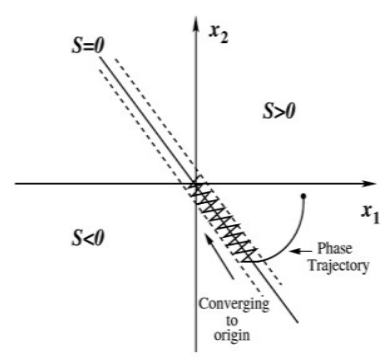

(b)
Fig. 2 Switching dynamics represented in phase plane for: (a) ideal sliding mode control and (b) practical sliding control mode (Reproduced from [8])

In contrast, the application of sliding mode methods in combination with observer control problems provide the ability to generate a sliding motion on the error between the measured plant output and the output of the observer such that to ensure that a sliding mode observer (SMO) produces a set of state estimates precisely matching with the actual output of the plant. Also the analysis of the average value of the applied observer injection signal, the so-called equivalent injection signal, contains useful information about the mismatch between the model used to define the observer and the actual plant. Furthermore, the discontinuous injection signals in this case don't remain anymore a drawback for software based observer frameworks in control applications [1], [2], [4], [5].

The development of SMO control strategy design in this research paper follows the same design guidelines suggested in [1],[2] and [4], [5] related to the design of sliding mode observer (SMO) control strategies for fault detection and isolation based on the equivalent injection signal principle.

\section{SLIDING MODE OBSERVER FOR LINEAR DC SERVOMOTOR WITHOUT MODEL OR DISTURBANCES UNCERTAINTIES}

For simulation purpose we assume in this section that the experimental values for the physical parameters of the dc servomotor are the same with those introduced in section II. With these values, the dynamics of the dc servomotor is described in state-space representation by the following first order differential equation:

$$
\begin{gathered}
\frac{d x}{d t}=A_{n \times n} x+B_{n \times m} u+D_{n \times q} \Psi(x, u, t) \\
y=C_{p \times n} x=\left[\begin{array}{ll}
1 & 0
\end{array}\right] x
\end{gathered}
$$

$$
A=\left[\begin{array}{cc}
-10 & 51.7 \\
-0.1034 & -2
\end{array}\right], B=\left[\begin{array}{l}
0 \\
2
\end{array}\right], D=\left[\begin{array}{l}
1 \\
0
\end{array}\right]
$$

$\Psi(x, u, t)=\left(-1000 T_{\text {Load }}\right)=-10 \sin (t)$.

where $x \in R^{n}$ is a $n$-dimensional state vector $(n=2), y \in$ $R^{p}$ is a $p$-dimensional output vector $(p=1)$, and $u \in R^{m}$ is a $m$-dimensional input vector $(m=1)$. For this development phase the load torque disturbance uncertainty is considered $T_{\text {Load }}=0$, (dc Servomotor no-load speed) and so $\Psi(x, u, t)=0$, in order to investigate in the first phase of our development only the linear case. By some manipulations of the matrices $A, B, C$ we can easily find that $B, C$ have a full rank and the pair $(A, C)$ is observable, as main requirements assumed in [1].

To design a sliding mode observer (SMO) for this control system we follow the same procedure as in [1]. Firstly we attach to the original system an Utkin observer [1], [2] in a canonical form. For this task we need to find a nonsingular state transform $T_{c} \in R^{n \times n}$ that changes the state vector $x$ in a state vector

$z=T_{c} x=\left[\begin{array}{l}z_{1} \\ z_{2}\end{array}\right], z_{1} \in R^{n-p}, z_{2} \in R^{p}, T_{c}=\left[\begin{array}{c}N_{c}^{T} \\ C\end{array}\right]$,

$N_{c} \in R^{n \times(n-p)}$

where the column of the matrix $N_{C}$ spans the null space of $C, \exists z_{1} \neq 0_{(n-p) \times 1} \stackrel{\text { yields }}{\longrightarrow} N_{c} \times z_{1}=0_{(n-p) \times 1}$.

This nonsingular state transform $T_{c}$ converts the nominal system (2) in the following canonical form:

$\frac{d z_{1}}{d t}=A_{11} z_{1}(t)+A_{12} z_{2}(t)+B_{1} u(t)$

$\frac{d z_{2}}{d t}=A_{21} z_{1}(t)+A_{22} z_{2}(t)+B_{2} u(t)$

Now the dynamics of the observer is described by the following similar equations:

$\frac{d \hat{z}_{1}}{d t}=A_{11} \hat{z}_{1}(t)+A_{12} \hat{z}_{2}(t)+B_{1} u(t)+L \vartheta$

$\frac{d \hat{z}_{2}}{d t}=A_{21} \hat{z}_{1}(t)+A_{22} \hat{z}_{2}(t)+B_{2} u(t)-\vartheta$

where the pair $\left(\hat{z}_{1}, \hat{z}_{2}\right)$ represent the estimated values of the transformed components state vector $z$, and $L \in R^{(n-p) \times p}$ is the observer gain matrix, given by

$\vartheta_{i}=\operatorname{Msgn}\left(\hat{z}_{2, i}-z_{2, i}\right), M \in R_{+}, i=1, \ldots, p$

The dynamics of the system errors is described by the following first order differential equations:

$\frac{d e_{1}}{d t}=A_{11} e_{1}(t)+A_{12} e_{2}(t)+L \vartheta$
$\frac{d e_{2}}{d t}=A_{21} e_{1}(t)+A_{22} e_{2}(t)-\vartheta$
$e_{1}(t)=\hat{z}_{1}(t)-z_{1}(t), e_{2}(t)=\hat{z}_{2}(t)-z_{2}(t)$

The observer gain matrix $L \in R^{(n-p) \times p}$ is chosen in order to make the spectrum of the matrix $\left(A_{11}+L A_{21}\right)$ to lie in $\mathrm{C}_{-}$, where the pair matrices $\left(A_{11}, A_{21}\right)$ is observable due to the fact that the pair $(A, C)$ is also observable. 
Without to lose the generality we can choose the coordinates transform matrix such as:

$$
T_{c}=\left[\begin{array}{c}
N_{c}^{T} \\
C
\end{array}\right]=\left[\begin{array}{ll}
0 & 1 \\
1 & 0
\end{array}\right]
$$

that converts the triple $(A, B, C)$ into $(\tilde{A}, \tilde{B}, \tilde{C})$, where the lines of the matrix $N_{c}^{T}$ span the null space of the vector $C$, and also:

$$
\begin{aligned}
& \tilde{A}=T_{c} A T_{c}^{-1}=\left[\begin{array}{cc}
-2 & -0.1034 \\
51.70 & -10
\end{array}\right]=\left[\begin{array}{ll}
A_{11} & A_{12} \\
A_{21} & A_{22}
\end{array}\right] \\
& \tilde{B}=T_{c} B=\left[\begin{array}{l}
2 \\
0
\end{array}\right]=\left[\begin{array}{l}
B_{1} \\
B_{2}
\end{array}\right], \tilde{C}=C T_{c}^{-1}=\left[\begin{array}{ll}
0 & 1
\end{array}\right]=\left[\begin{array}{ll}
C_{1} & C_{2}
\end{array}\right]
\end{aligned}
$$

From the matrix structure $\tilde{A}$ we get $A_{11}=-2$ (stable), and $A_{12}=-0.1034, A_{21}=51.70, A_{22}=-10$. Also, $B_{1}=2, B_{2}=0, C_{1}=0, C_{2}=1$.

The value of the observer matrix gain $L$ can be choose such as $A_{11}+L A_{21}<0$, let take this $L=-1<-\frac{A_{11}}{A_{21}}=$ 0.0387. Setting the observer matrix gain $L$ to 0.01 the dynamics of the linear observer and of its error are described by the following first order differential equations:

$$
\begin{aligned}
& \frac{d \hat{z}_{1}}{d t}=-2 \hat{z}_{1}(t)-0.1034 \hat{z}_{2}(t)+2 u(t)-\vartheta \\
& \frac{d \hat{z}_{2}}{d t}=51.70 \hat{z}_{1}(t)-10 \hat{z}_{2}(t)-\vartheta \\
& \frac{d e_{1}}{d t}=-2 e_{1}(t)-0.1034 e_{2}(t)+0.01 \vartheta \\
& \frac{d e_{2}}{d t}=51.70 e_{1}(t)-10 e_{2}(t)-\vartheta \\
& \vartheta=\operatorname{sgn}\left(\hat{z}_{2}-z_{2}\right)=\operatorname{sgn}\left(e_{2}(t)\right), M=1
\end{aligned}
$$

\subsection{SIMULATION RESULTS}

In all the above equations we set $u(t)=12[\mathrm{~V}]$ in order to solve the problem of designing a sliding mode observer (SMO) by using MATLAB/SIMULINK software package. The model of the original system in SIMULINK is shown in figure 3 , and the evolution of the states, i.e. angular $\operatorname{speed}\left(x_{1}\right)$ and armature current $\left(x_{2}\right)$ are shown in figures 4 and 5 . The state-space representation model of the dc servomotor in canonical form and the Utkin classical observer dynamics model embedded in the same integrated control structure are represented in MATLAB/SIMULINK, as are shown in figure 6 and the evolution of the both model and estimated states, namely the angular speed $\left(z_{2}\right)$ and the armature current $\left(z_{1}\right)$ are shown in figures 7 and 8 . The dc servomotor angular speed $\left(e_{2}\right)$ and armature currents $\left(e_{1}\right)$ SMO residuals are shown in figures 9 and 10 . In figure 11 are shown the SMO errors dynamics modeled in SIMULINK.

An ideal sliding motion will take place on the sliding surface [1], [2], [4], [5]:

$$
\left.S_{w}=\left\{\left(e_{1}, e_{2}\right) \mid e_{2}=0\right)\right\}
$$

After some finite time $t_{s}$, for all subsequent time, $e_{2}=0$, and $\frac{d e_{2}}{d t}=0$.

The corresponding sliding mode dynamics are given in [1]-[2]:

$$
\frac{d \tilde{e}_{1}(t)}{d t}=\tilde{A}_{11} \tilde{e}_{1}(t)
$$

where

$$
\begin{aligned}
& \tilde{e}_{1}(t)=e_{1}(t)+L e_{2}(t)=e_{1}(t)-e_{2}(t) \\
& \tilde{A}_{11}=A_{11}+L A_{21}=-2-51.70=-53.70<0 .
\end{aligned}
$$

Since $\tilde{A}_{11}<0$, the linear homogenous equation (12) has a stable solution, $\tilde{e}_{1}(t)=C_{0} e^{-53.70 t}$, where $C_{0}$ is a integration constant determined from the initial condition $\tilde{e}_{1}(0)=\tilde{e}_{10}$. By a suitable choice of the gain $L$, such as in our case study $L=-1<0.0387$,we can conclude that always the system is stable, therefore $\tilde{e}_{1}(t) \stackrel{\text { yields }}{\longrightarrow} 0$, and also $\hat{z}_{1}(t) \stackrel{\text { yields }}{\longrightarrow} z_{1}(t)$ as $t \stackrel{\Delta}{\rightarrow} \infty$.

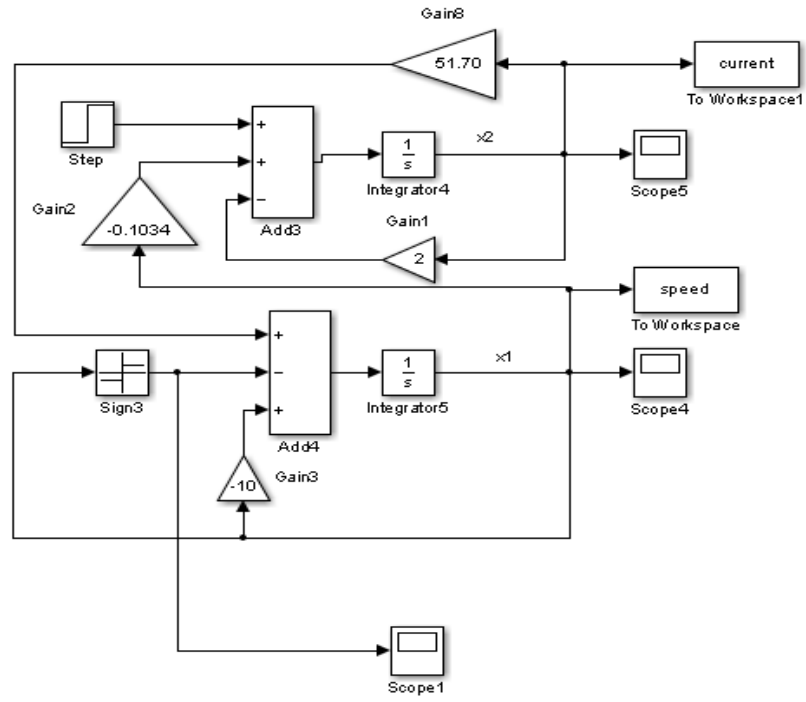

Fig. 3 DC servomotor state space-representation of nominal model in MATLAB/SIMULINK

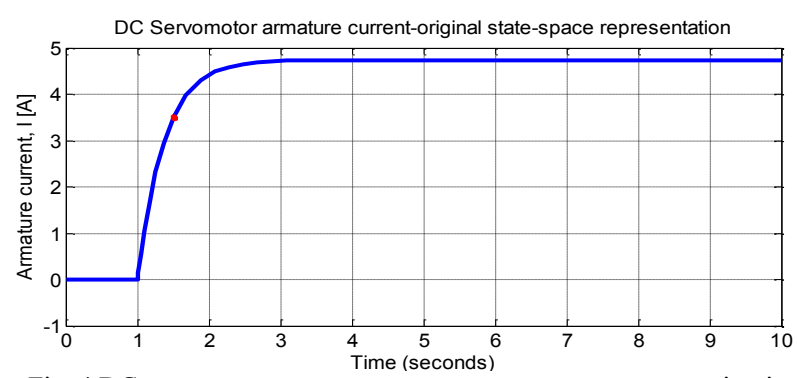

Fig. 4 DC servomotor armature current-state-space representation in MATLAB/SIMULINK

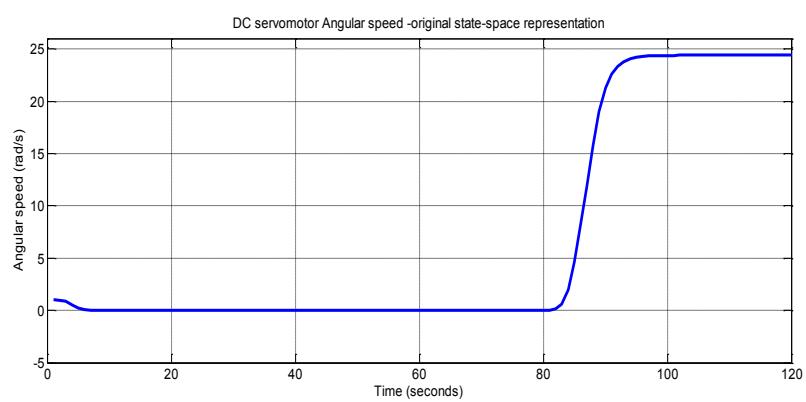

Fig. 5 DC servomotor angular speed- original state-space representation model in MATLAB/SIMULINK 
In figure 12 is captured much more commutations around the sliding line:

$$
S_{w}: \frac{d e_{2}}{d t}=0, e_{2}=0
$$

and is shown the time switching control function

$\vartheta=\operatorname{sgn}\left(\hat{z}_{2}-z_{2}\right)=\operatorname{sgn}\left(e_{2}(t)\right), M=1, L=-1$.

Secondly, we build a classical Utkin observer with linear injection that is used in next section to introduce a constructive sliding mode observer design framework.

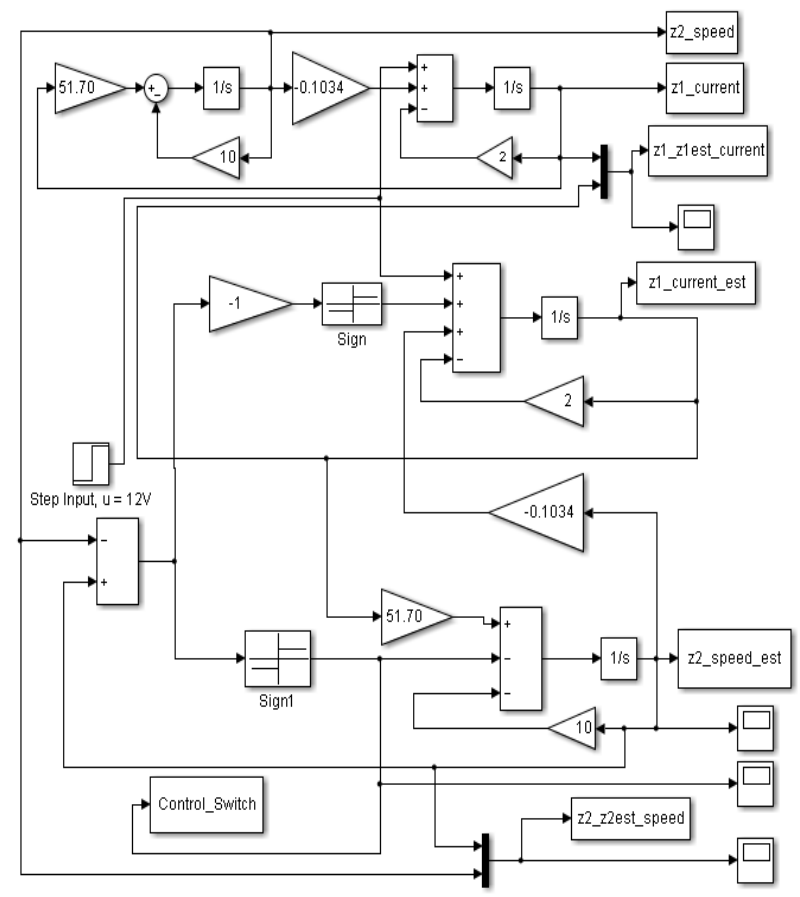

Fig. 6 Sliding Mode Observer - state space-representation in MATLAB/SIMULINK

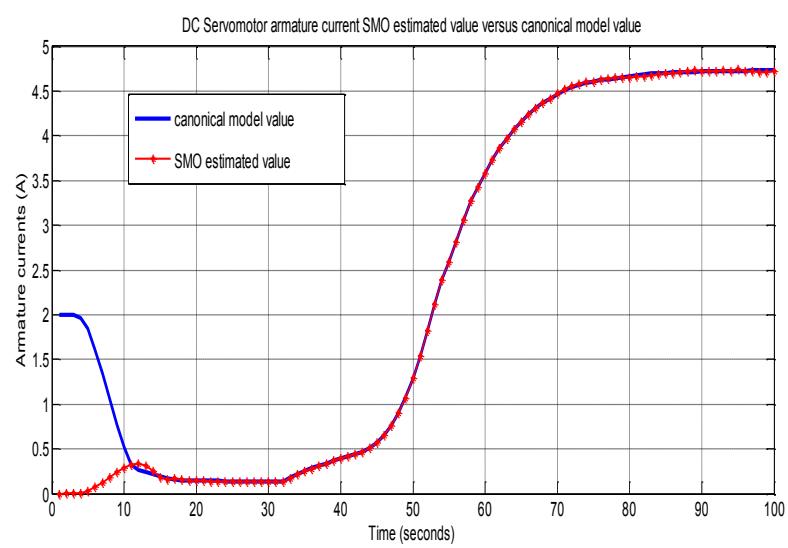

Fig. 7 DC servomotor armature current estimated versus canonical model current using SMO control strategy in MATLAB/SIMULINK

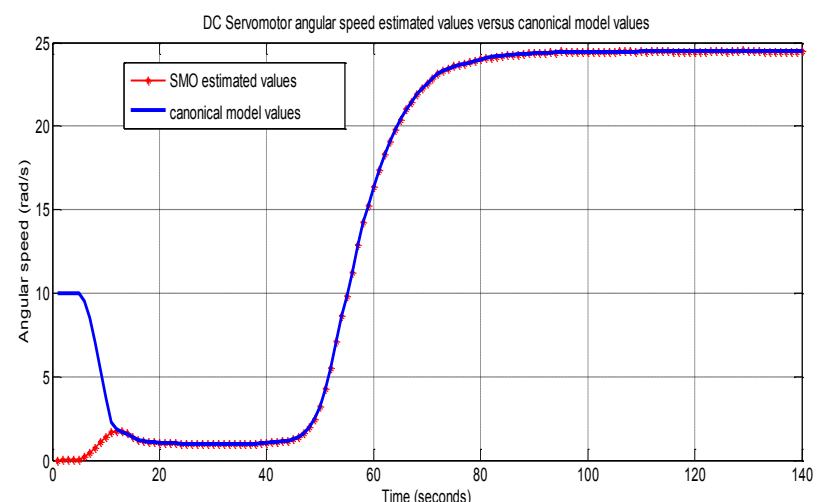

Fig. 8 DC servomotor angular speed estimated versus model angular speed using SMO control strategy in MATLAB/SIMULINK

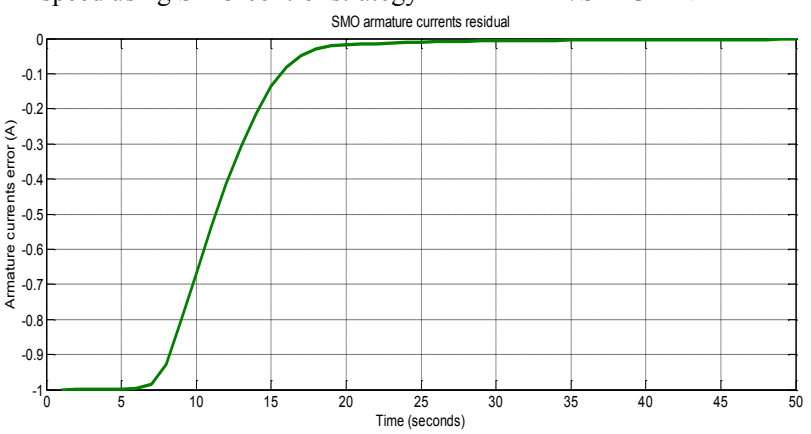

Fig. 9 DC servomotor SMO armature currents residual

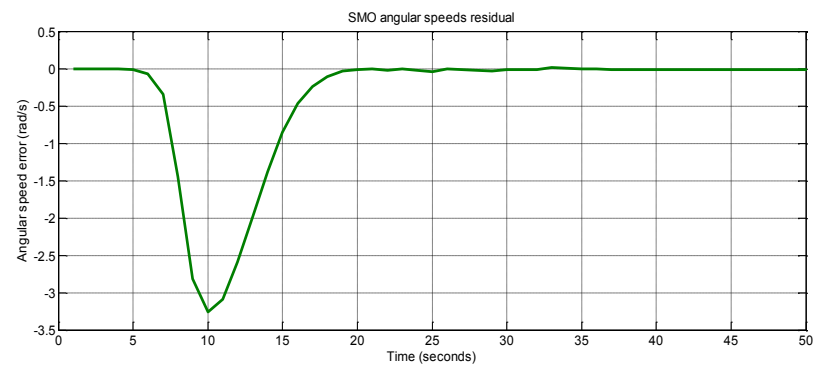

Fig. 10 DC servomotor angular residual speed using SMO control strategy

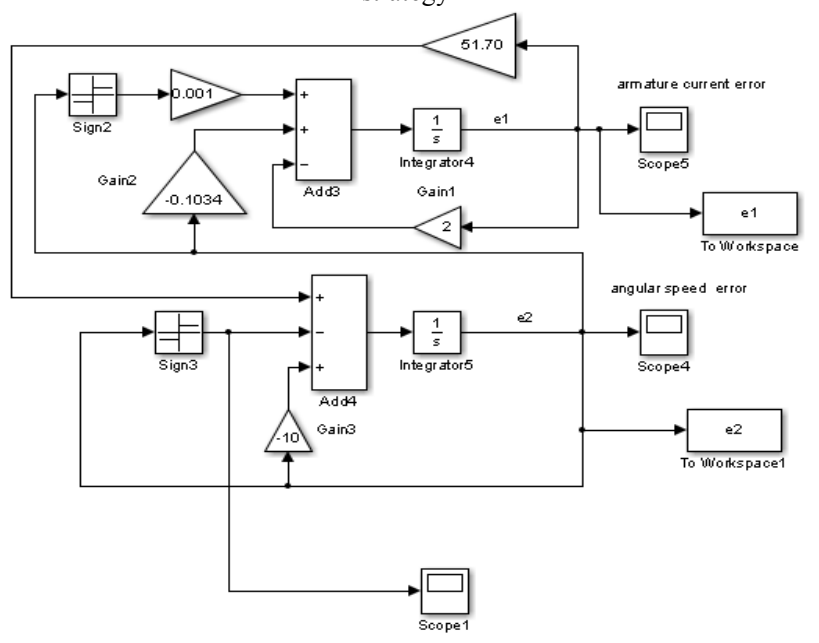

Fig. 11 DC servomotor SMO error dynamics - SIMULINK model 


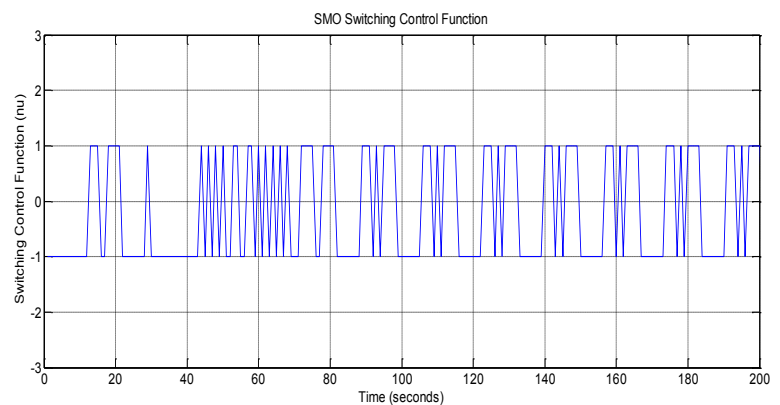

Fig. 12 SMO control switching function around sliding line

\section{V.SLIDING MODE OBSERVER FOR LINEAR DC SERVOMOTOR WITH MODEL OR DISTURBANCE UNCERTAINTY}

For the uncertain system (2) we define the following observer described in state-space representation [1]-[2], [4][5] by the following equations:

$$
\frac{d \hat{x}}{d t}=A_{n \times n} \hat{x}+B_{n \times m} u-G_{L, n \times p} C_{p \times n} e(t)+G_{N, n \times p} \vartheta
$$

where the error $e(t)$ is

$$
e(t)=\hat{x}(t)-x(t)
$$

$\vartheta$ is a discontinuous switching function about the sliding hyperplane:

$\left.S_{w}=\left\{e \in R^{n} \mid C e=0\right)\right\}$

and the precise structure of injection signal matrices gains $G_{L, n \times p}, G_{N, n \times p}$ is to be determined.

The existence conditions for a SMO of the form (13) that rejects the uncertainty class $\Psi(x, u, t): R_{+} \times R^{n} \times R^{m} \rightarrow$ $R^{n}$ of dc servomotor load torque $T_{\text {Load }}=$ $10 \operatorname{sint}(t)$ described in (2) are given in [1]-[2], [4]-[5]:

- $\operatorname{rank}(C \times D)=q=1,1=q \leq p<n=2$

- any invariant zeros of the original nominal system matrices $(A, D, C)$ given in (2) must lies in the half left complex plane $C_{-}$(stable).

For a square nominal linear system these two conditions require that the triplet $(A, D, C)$ to be relative degree one and minimum phase. Furthermore, the existence conditions depend upon a specific selection of the uncertainty channel and the observer design will be directly determined by the uncertainty distribution matrix $D$. The necessary and sufficient conditions for existence of a sliding mode observer (SMO) together with the canonical form provide a pathway to a constructive method for observer design [2], [4], [5].

The original nominal system (2) is converted in the canonical form (3) using a possible change in coordinates $z_{c}=T_{c} x$ through a canonical transformation matrix $T_{c}$ given in (8):

$$
\begin{aligned}
& A_{c}=T_{c} A T_{c}^{-1}=\left[\begin{array}{cc}
-2 & -0.1034 \\
51.70 & -10
\end{array}\right]=\left[\begin{array}{ll}
A_{11 c} & A_{12 c} \\
A_{21 c} & A_{22}
\end{array}\right] \\
& B_{c}=T_{c} B=\left[\begin{array}{l}
2 \\
0
\end{array}\right]=\left[\begin{array}{l}
B_{1 c} \\
B_{2 c}
\end{array}\right] \\
& C_{c}=C T_{c}^{-1}=\left[\begin{array}{ll}
0 & 1
\end{array}\right]=\left[\begin{array}{ll}
C_{1 c} & C_{2 c}
\end{array}\right] \\
& D_{c}=T_{c} D=\left[\begin{array}{ll}
0 & 1 \\
1 & 0
\end{array}\right]\left[\begin{array}{l}
1 \\
0
\end{array}\right]=\left[\begin{array}{l}
0 \\
1
\end{array}\right]=\left[\begin{array}{l}
D_{1 c} \\
D_{2 c}
\end{array}\right]
\end{aligned}
$$

In this structure all the required existence conditions mentioned in [2] to design a SMO are satisfied. The matrix gain $\bar{L}$ defined in [Sarah] for our case study becomes $\tilde{L}=$ $\bar{L}=0$.

Define now a new nonsingular matrix $T_{L}$ of the following structure [2], [4], [5]:

$T_{L}=\left[\begin{array}{cc}I_{n-p} & \bar{L} \\ 0 & D_{2}\end{array}\right]=\left[\begin{array}{ll}1 & 0 \\ 0 & 1\end{array}\right]$

that converts the system from canonical form (17) in the following state-space representation:

$z_{L}=T_{L} x_{c}$

$$
\begin{aligned}
A_{L} & =T_{L} A_{c} T_{L}^{-1}=A_{c}=\left[\begin{array}{cc}
-2 & -0.1034 \\
51.70 & -10
\end{array}\right] \\
A_{L} & =\left[\begin{array}{ll}
A_{11, L} & A_{12, L} \\
A_{21, L} & A_{22, L}
\end{array}\right] \\
B_{L} & =T_{L} B_{c}=\left[\begin{array}{l}
2 \\
0
\end{array}\right]=\left[\begin{array}{l}
B_{1, L} \\
B_{2, L}
\end{array}\right], \\
C_{L} & =T_{L}^{-1} C_{c}=C_{c}=\left[\begin{array}{ll}
0 & 1
\end{array}\right]=\left[\begin{array}{ll}
C_{1, L} & C_{2, L}
\end{array}\right] \\
D_{L} & =T_{L} D_{c}=\left[\begin{array}{ll}
1 & 0 \\
0 & 1
\end{array}\right]\left[\begin{array}{l}
0 \\
1
\end{array}\right]=\left[\begin{array}{l}
0 \\
1
\end{array}\right]=\left[\begin{array}{l}
D_{1, L} \\
D_{2, L}
\end{array}\right],
\end{aligned}
$$

and so, by chance, the same structure as in the canonical form.

The system triplet $\left(A_{L}, C_{L}, D_{L}\right)$ can be put in the following form [1], [2]:

$$
\begin{aligned}
\frac{d z_{1, L}}{d t}= & A_{11, L} z_{1, L}(t)+A_{12, L} z_{2, L}(t)+B_{1, L} u(t) \\
\frac{d z_{2, L}}{d t}= & A_{21, L} z_{1, L}(t)+A_{22, L} z_{2, L}(t)+B_{2, L} u(t)+\cdots \\
& D_{2, L} \Psi(x, u, t)
\end{aligned}
$$

$y(t)=C_{2, L} z_{2, L}(t)=z_{2, L}(t)$,

and so we can write also the following equivalent equation:

$\frac{d y(t)}{d t}=A_{21, L} z_{1, L}(t)+A_{22, L} y(t)+B_{2, L} u(t)+\cdots$

$$
D_{2, L} \Psi(x, u, t)
$$

The corresponding observer is described by the following equations:

$$
\begin{aligned}
\frac{d \hat{z}_{1, L}}{d t}= & A_{11, L} \hat{z}_{1, L}(t)+A_{12, L} \hat{y}(t)+B_{1, L} u(t)-A_{12, L} e_{y}(t) \\
\frac{d \hat{y}(t)}{d t}= & A_{21, L} z_{1, L}(t)+A_{22, L} \hat{y}(t)+B_{2, L} u(t)-\cdots \\
& \left(A_{22, L}-A_{22, L}^{s}\right) e_{y}(t)+\vartheta
\end{aligned}
$$$$
e_{2, L}(t)=\hat{z}_{2, L}(t)-z_{2, L}(t)=\hat{y}(t)-y(t)=e_{y}(t)
$$

where $A_{22, L}^{S}$ is a stable design matrix, let us to take it $A_{22, L}^{S}=-2$. Let us to consider also a symmetric positive definite matrix for $A_{22, L}^{S}, \quad P_{2} \in R^{p \times p}$, that is a unique solution of the Lyapunov equation:

$\left(A_{22, L}^{S}\right)^{T} P_{2}+P_{2}\left(A_{22, L}^{S}\right)=-Q_{2}$

with $Q_{2} \in R^{p \times p}$ a symmetric positive definite design matrix.

For a particular selection of matrix $Q_{2}=4$ we get:

$-4 P_{2}=-4 \rightarrow P_{2}=1$

Now a robust observer design is well defined and can be described by the following equations:

$\frac{d \hat{x}}{d t}=A_{L_{n \times n}} \hat{x}+B_{L_{n \times m}} u-G_{L_{-} L, n \times p} C_{L_{p \times n}} e(t)+G_{N_{-} L, n \times p} \vartheta$
$\hat{y}(t)=C_{L_{p \times n}} \hat{x}(t)$
$e(t)=\hat{x}(t)-x(t)$
$e_{y}(t)=\hat{y}(t)-y(t)$ where the gain matrices have the particular form [1], [2],[4], [5]: 
$G_{L_{-} L, n \times p}=T_{c}^{-1}\left[\begin{array}{c}A_{12, L} \\ A_{22, L}-A_{22, L}^{S}\end{array}\right]=\left[\begin{array}{c}-8 \\ -0.10348\end{array}\right]$

$G_{N_{-} L, n \times p}=\left\|D_{2}\right\| T_{c}^{-1}\left[\begin{array}{c}0 \\ I_{p}\end{array}\right]=\left[\begin{array}{l}1 \\ 0\end{array}\right]$

Corresponding to a particular selection of canonical coordinates transform, $T_{c}=\left[\begin{array}{ll}0 & 1 \\ 1 & 0\end{array}\right]$.

Also, the discontinuous switching function $\vartheta$ about the sliding hyperplane $S_{w}$ (15) is given by:

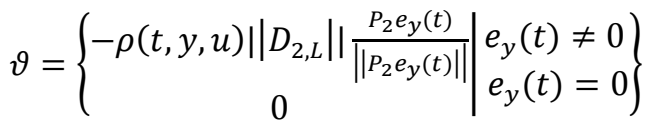

Remark: A big advantage of this development in this formulation of the sliding mode observer (SMO) design framework is that there is no requirement for $(\mathrm{A}, \mathrm{C})$ to be observable [2].

On the state components the developed robust observer given in (26) can be written in the following form:

$\frac{d \hat{x}_{1}}{d t}=-2 \hat{x}_{1}(t)-0.1034 \hat{y}(t)+2 u(t)+8 e_{y}(t)-\cdots$

$\rho(t, y, u) \operatorname{sign}\left(e_{y}(t)\right)$

$\frac{d \hat{y}(t)}{d t}=51.70 \hat{x}_{1}(t)-10 \hat{y}(t)+0.1034 e_{y}(t)$

with the dynamical errors described by following equations:

$\frac{d e_{1}(t)}{d t}=A_{11, L} e_{1}(t)=-2 e_{1}(t)$

$\frac{d e_{y}(t)}{d t}=A_{21, L} e_{1}(t)+A_{22, L}^{s} e_{y}(t)+\vartheta-D_{2, L} \Psi(x, u, t)$ $=51.70 e_{1}(t)-2 e_{y}(t)+\vartheta-\Psi(x, u, t)$

$e_{1}(t)=\hat{x}_{1}(t)-\hat{x}_{1}(t)$

$e_{y}(t)=\hat{y}(t)-y(t)$

where the uncertainty function $\rho(t, y, u)$ is bounded by: $\rho(t, y, u) \geq r|| u(t) \|+\alpha(y, t)+\delta \geq 12 r+10 \sin (t)+\delta$ For a particular selection according to [Sarah]:

$r=0.7>0, \delta=1.6>0, \alpha(y, t)=-10 \sin (t)$,

the equations become:

$\frac{d \hat{x}_{1}}{d t}=-2 \hat{x}_{1}(t)-0.1034 \hat{x}_{2}(t)+2 u(t)+8 e_{y}(t)-$

$\ldots-20 \operatorname{sign}\left(e_{y}(t)\right)$

$\frac{d \hat{y}(t)}{d t}=51.70 \hat{x}_{1}(t)-10 \hat{y}(t)+0.1034 e_{y}(t)$

where the scalar function $\rho(t, y, u)$ is bounded by:

$\rho(t, y, u) \geq 20$

\subsection{SIMULATION RESULTS}

The dynamics errors of the Sliding mode Observer model (30) attached to the dc Servomotor actuator with disturbance uncertainty (the load torque, $T_{\text {Load }}=10 \operatorname{sint}(t)$ ) are modeled in SIMULINK and shown in figure 13. Their dynamic evolution, i.e. armature current residual $\left(e_{1}\right)$ and angular speed residual $\left(e_{y}\right)$, is shown in figures 14 and 15 .

The SMO control switching function around sliding line $\vartheta$ is calculated according to (28) and (31) and is shown in figure 16. The state-space representation of the robust Sliding Mode Observer model (26) in canonical form is represented also in SIMULINK, and the evolution of the both model and estimated states, namely the angular output speed $(y(t), \hat{y}(t))$ and the armature current $\left(x_{1}(t), \hat{x}_{1}(t)\right)$ are shown in figures 17 and 18, for open-loop system for the same setting $u(t)=12[\mathrm{~V}]$. From the last two figures we observe that after approximately 2 seconds, visually perfect replication of the true and estimated states is taking place. To demonstrate the robustness of the nonlinear observer tracking the output from the dc Servomotor when the initial conditions of the true states and observer states are deliberately set to different values. If the nonlinear component is removed by setting $\rho(t, y, u)$ to zero, the resulting Luenberger Observer [2] behaves as simple observer without sliding motion.

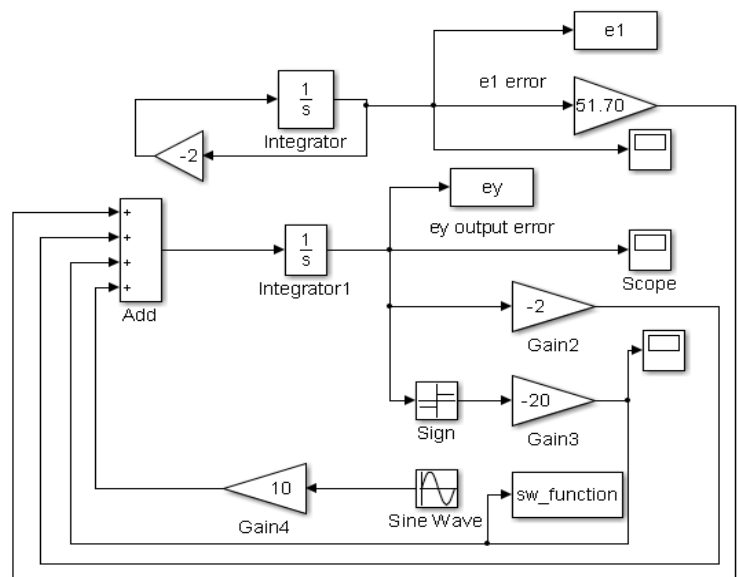

Fig. 13 DC servomotor SMO error dynamics with modeling uncertainty SIMULINK model

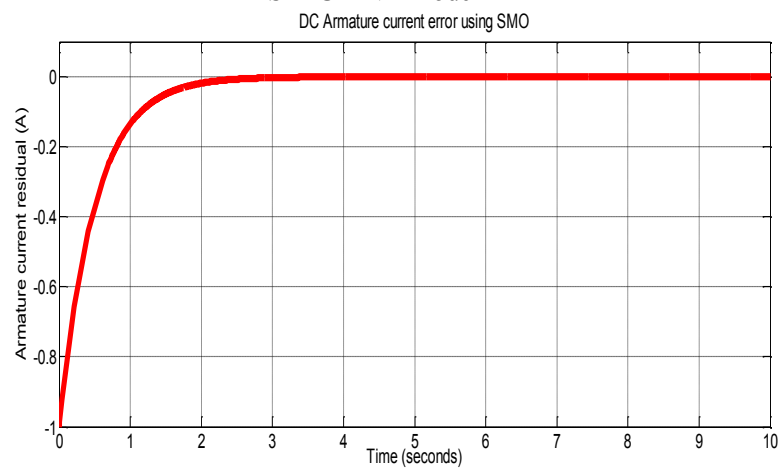

Fig. 14 DC servomotor armature residual current using SMO control strategy with modeling uncertainty

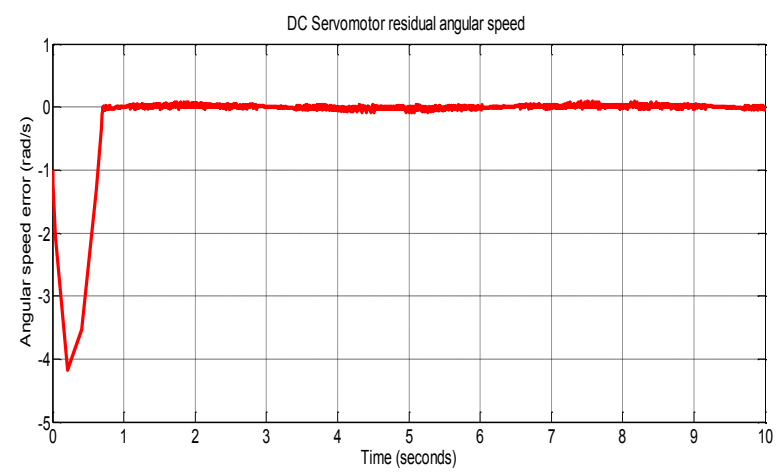

Fig. 15 DC servomotor angular residual speed using SMO control strategy with modeling uncertainty 


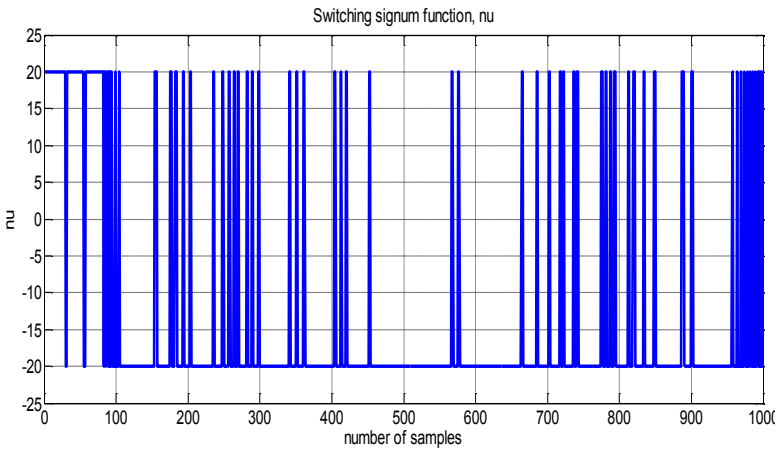

Fig. 16 SMO control switching function around sliding line

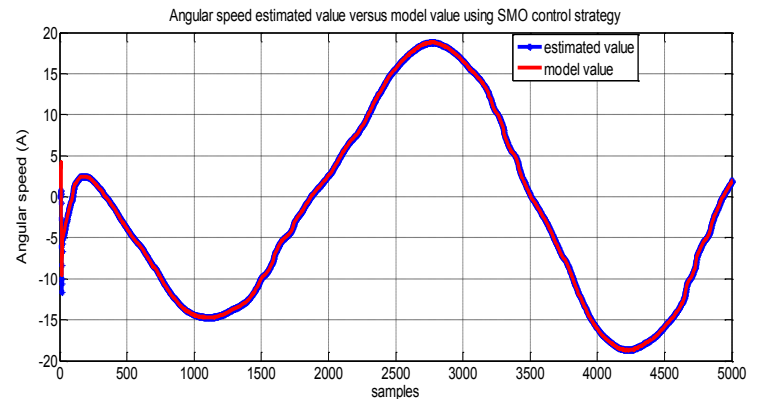

Fig. 17 DC servomotor angular speed estimated versus model angular speed using SMO control strategy with modeling uncertainty MATLAB/SIMULINK simulations

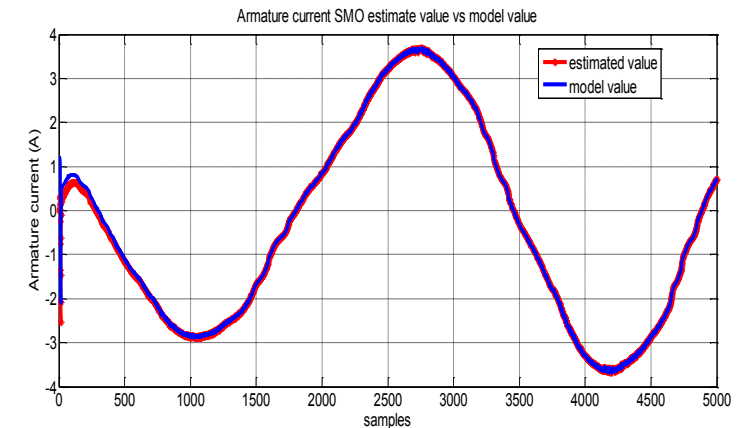

Fig. 18 DC servomotor armature current estimated versus model value using SMO control strategy with modeling uncertainty MATLAB/SIMULINK simulations

\section{SLIDING MODE OBSERVER REAL-TIME IMPLEMENTATION}

In control systems literature rarely we find details about the real-time software and hardware implementation aspects, and no sufficient attention is given about the algorithms and the sampling time selection. Usually the implementation aspect and real-time control systems design are connected together but in the most cases this connection is always ignored. Furthermore the real-time control systems design is treated from control perspective ignoring the implementation aspects of the control algorithms. Fortunately, recently the real-time implementation and design aspects get a considerable attention from part of control engineering community due to the introduction of new software tools like MATLAB/SIMULINK with its RTW (Real-Time Workshop) and the RTWT (Real-Time Windows Target) Toolboxes. The real-time platform used to perform these real-time simulations is a MATLAB R2013a with SIMULINK running on two processors WINDOWS OS machine. Certainly these new real-time platforms do the implementation of real-time experiments easier and save much time but on the other hand they have some drawbacks regarding a good perception of the real-life problems that could appear during the real-time implementation of the control systems.

\section{CONCLUSIONS}

In this paper, we have studied the possibility of using a Sliding Mode Observer strategy design to a dc Servomotor actuator with disturbance uncertainty that is integrated in the same control system structure. The implementation in real time of SMO proposed control strategy will be very useful for our future developments in fault detection and isolation (FDI) control applications based on the equivalent signal injection principle [1]-[4]. This new FDI control strategy will be design in the future work based on the injection signal principle [1]-[2], [4]-[5]. The main contributions in our research are summarized briefly as follows:

(a) Comparison of performance capabilities and advantages of real-time implementation of a Sliding Mode Observer (SMO) versus Sliding Mode Control (SMC),

(b) Implementation in real time a Sliding Mode Observer of a linear dc Servomotor actuator without uncertainty,

(c) Implementation in real time a Sliding Mode Observer for a linear dc Servomotor actuator with bounded disturbance uncertainty.

\section{REFERENCES}

[1]. Sarah K. Spurgeon, "Sliding Mode Observers - historical background and basic introduction", Spring School, Aussois, June 2015.

[2]. Sarah K. Spurgeon, "Sliding Mode Observers - toward a constructive design framework", Spring School, Aussois, June 2015.

[3]. A. Aref, T. Tran, "Using Fuzzy Logic and Q-Learning for Trust Modeling in Multi-agent Systems", Proceedings of the 2014 Federated Conference on Computer Science and Information Systems, Warsaw, Poland, ACSIS, Vol. 2, pp. 59-66, 2014, DOI: $10.15439 / 2014 \mathrm{~F} 482$.

[4]. H. K. Khalil, L. Praly, "High-gain observers in nonlinear feedback control", Int. J. Robust. Nonlinear Control, vol.24, issue 6, pp.993-1015, John Wiley \& Sons, Ltd, 2013, DOI: $10.1002 / \mathrm{rnc} .3051$

[5]. X.G. Yan, C. Eduards, "Nonlinear robust fault reconstruction and estimation using a sliding mode observer", Elsevier, ScienceDirect, Automatica, vol. 43, pp.1605 - 1614, 2007, DOI:10.1016/j.automatica.2007.02.008.

[6]. Amira Sayed A. Aziz, Ahmad Taher Azar, Mostafa A. Salama, Aboul Ella Hassanien, Sanaa El-Ola Hanafy, "Genetic Algorithm with Different Feature Selection Techniques for Anomaly Detectors Generation", Proceedings of the 2013 Federated Conference on Computer Science and Information Systems, Krakow, Poland, pp. 769-774, 2013.

[7]. N.Tudoroiu, K. Khorasani, "Satellite Fault Diagnosis using a Bank of Interacting Kalman Filters", IEEE Transactions on Aerospace and Electronic Systems, vol. 43, no.4, 2007, pp. 1334-1350, IEEEXplore, DOI: 10.1109/TAES.2007.4441743.

[8]. E-R. Tudoroiu, "Conceiving and Implementing Applications using Real-Time UML", PhD Thesis, Cluj-Napoca Technical University, Romania, 2012.

[9]. Control Tutorials for MATLAB, Carnegie Mellon Lab, University of Michigan, http://ctms.engin.umich.edu. 